\title{
Reflets
}

Revue d'intervention sociale et communautaire

\section{Défier les attentes : résurgence du mouvement pour une université franco-ontarienne}

\section{Geneviève L. Latour et C.-Isabelle Gagnon}

Volume 23, numéro 2, automne 2017

URI : https://id.erudit.org/iderudit/1043307ar

DOI : https://doi.org/10.7202/1043307ar

Aller au sommaire du numéro

Éditeur(s)

Reflets, Revue d'intervention sociale et communautaire

ISSN

1203-4576 (imprimé)

1712-8498 (numérique)

Découvrir la revue

Citer cet article

Latour, G. L. \& Gagnon, C.-I. (2017). Défier les attentes : résurgence du mouvement pour une université franco-ontarienne. Reflets, 23(2), 206-217.

https://doi.org/10.7202/1043307ar d'utilisation que vous pouvez consulter en ligne. 


\title{
Défier les attentes : résurgence du mouvement pour une université franco-ontarienne
}

\author{
Geneviève L. Latour \\ Originaire de St-Albert dans l'Est ontarien \\ Candidate à la maîtrise en travail social, Université de Moncton \\ Cofondatrice et ancienne coprésidente du REFO \\ C.-Isabelle Gagnon \\ Née à Vanier, un quartier d'Ottawa en Ontario \\ Baccalauréat en études des fermmes et service social et \\ Maîtrise en service social, Université d'Ottawa \\ Ancienne coprésidente du RÉFO
}

\section{Introduction}

Le mouvement militant pour une université franco-ontarienne a pris un nouveau souffle et évolue depuis quatre ans. L'article porte sur la trajectoire suivie par les organismes grassroots jeunesse et étudiants qui mènent principalement le dossier. Ils ont su oser, revendiquer et mobiliser d'une manière qui s'est rarement vue en Ontario français depuis le XXI ${ }^{\mathrm{e}}$ siècle. Nous traitons des forces et des défis rencontrés en milieu communautaire minoritaire et des réponses qui leur ont été apportées. Enfin, nous dressons une appréciation qualitative des changements sociaux engendrés par cette lutte.

\section{Mise en contexte}

Le dossier de la création d'une université franco-ontarienne date des années 1950. Les luttes menées au tournant des années 1980 et 1990 ont été chapeautées principalement par des membres de la communauté académique. Puis, avec l'annonce de la création des collèges de langue française dans le Nord et dans le Sud (1993) de la province, le projet de création d'une université franco-ontarienne a été mis de côté. Il est vrai qu’au début des années 2000, la Fédération de la jeunesse franco-ontarienne (FESFO) a publié un 
mémoire lançant un appel au gouvernement provincial de mettre sur pied une université unilingue francophone en Ontario (Fédération de la jeunesse franco-ontarienne, 2004). Cependant, ce n'est qu'après la création du Regroupement étudiant franco-ontarien (RÉFO) en $2009^{1}$ que le projet reprend véritablement de l'intérêt.

À l'automne 2012, lors de sa III e assemblée générale, le RÉFO reçoit le mandat de mener des États généraux sur le postsecondaire en Ontario français. L'organisme avait reçu une certaine attention médiatique pour avoir porté le dossier de réinstauration de la bourse d'études en français ${ }^{2}$. De plus, dans la province voisine, le mouvement étudiant québécois a eu une influence considérable sur la population quand est venu le temps d'aller aux urnes. Le Québec venait tout juste de sortir de son "printemps érable ", c'était la rentrée et la crise étudiante attirait encore beaucoup l'attention des médias et du gouvernement.

Les jeunes, les étudiantes et les étudiants avaient un momentum à garder et ont saisi une série d'occasions de se faire entendre. Le dossier de création d'une université francoontarienne est donc revenu dans la sphère médiatique et politique. Ce qui était différent cette fois-ci, c'est que le mouvement était mené par et pour la jeunesse et la population étudiante (FESFO et RÉFO), avec la communauté (Assemblée de la francophonie de l'Ontario). En 2013 ont eu lieu des consultations provinciales auxquelles ont participé plus de 800 membres de l'ensemble de la communauté dans dix villes de l'Ontario. Le processus s'est terminé en 2014 par un sommet provincial. Pendant ce temps, les porteparoles des trois organismes partenaires ont tenu des dizaines de rencontres politiques avec des ministres et des députés. Sans compter qu'il y a eu plus d'une centaine d'articles écrits et d'entrevues données, et ce, à l'échelle provinciale.

À l'hiver 2015, une demande officielle a été déposée à la première ministre de l'Ontario, lui demandant de s'engager à créer cette université. À l'automne de la même année, des porte-paroles de la FESFO, du RÉFO et de l'Assemblée de la francophonie de l'Ontario ont rencontré une vingtaine de députés des trois partis politiques à l'Assemblée législative dans le cadre d'une campagne de sensibilisation au projet de création d'une université de langue française dans la province. Plus tard, à la mi-novembre, le projet de loi 104 "Loi constituant l'Université de l'Ontario français ", déposé par le NPD, a été adopté en deuxième lecture. Finalement, le 18 février 2016 s'est tenue la Journée d'action provinciale pour une université franco-ontarienne. 


\section{Les forces}

\section{Rôle de direction joué par les jeunes}

Dès l'automne 2012, le Regroupement a su mobiliser des étudiantes et étudiants de partout en province. Bien que les jeunes soient souvent perçus comme apathiques et désintéressés de la politique, le RÉFO a démontré qu'ils "sont toujours politisés, mais peut-être autrement; ils continuent d'être engagés, mais peut-être ailleurs que là où les chercheurs regardent habituellement»(Gallant et Garneau, 2016, p. 2).

Il est donc primordial que les jeunes fassent partie des campagnes à l'égard de l'avenir de la francophonie ontarienne. Gauthier et Piché (2001, p. 81) expliquent que " [1]es jeunes portent bien souvent un regard neuf sur le monde dans lequel ils entrent et sont à ce titre susceptibles d'apporter un éclairage novateur sur des réalités complexes ». Deux des trois partenaires du projet sont des organismes jeunesse et étudiants. Leur participation au mouvement pour une université franco-ontarienne est une de ses forces. Le RÉFO et la FESFO ont su reconnaître le rôle important que jouent les jeunes, et les jeunes ont su se reconnaître dans ces organismes " par et pour » et se sont ainsi sentis interpellés par ce projet revendicateur.

\section{Portée provinciale}

Le processus des États généraux sur le postsecondaire en Ontario français a permis au RÉFO, à la FESFO et à l'Assemblée de la francophonie de l'Ontario de consulter la communauté franco-ontarienne pendant deux ans. En octobre et novembre 2013, six consultations ouvertes à toute la communauté ont regroupé 550 personnes à Windsor, Toronto, Ottawa, Sudbury, Timmins et Thunder Bay; quatre consultations jeunesse ont rassemblé 250 jeunes du secondaire à Windsor, Kingston, Mattawa et Cochrane (États généraux sur le postsecondaire en Ontario français, 2014). Le tout s'est terminé par le Sommet provincial en octobre 2014 à Toronto, rassemblant plus de 150 personnes (États généraux sur le postsecondaire en Ontario français, 2015).

Ce processus de consultation a "permis aux membres de la communauté francoontarienne de partager leurs idées et aspirations pour l'avenir du système postsecondaire de langue française et d'établir des priorités de la communauté d'ici les dix prochaines années en ce qui concerne l'enseignement supérieur en Ontario français » (États généraux sur le postsecondaire en Ontario français, 2013a). Il a également permis de sensibiliser et mobiliser la communauté francophone sur l'ensemble du territoire ontarien. 


\section{Information et prise de conscience}

Lors du processus des États généraux, plusieurs études et statistiques ont été partagées avec la communauté dans toute la province. En plus d'obtenir ce portrait statistique, objectif et factuel, les organisatrices et organisateurs des États généraux ont reconnu l'importance de prendre en considération le regard et le vécu des personnes concernées afin de coconstruire la réalité des études postsecondaires en Ontario français.

Les consultations ont permis de réconcilier les savoirs scientifiques d'experts et les savoirs expérientiels des personnes touchées par la situation. Lavoie et Panet-Raymond (2014, p. 101) expliquent l'importance d'une telle analyse de la situation : « une étape de l'intervention qui favorise l'empowerment communautaire dans la mesure où elle renforcera, chez les personnes et les collectivités engagées, une prise de conscience de leurs propres capacités à déclencher et à contrôler l'action en valorisant un rapport actif aux savoirs et à la réalité ". Ces deux années de consultations ont donc permis à la communauté franco-ontarienne, dans sa diversité, de définir l'enjeu et de se l'approprier.

\section{Utilisation d'une diversité de stratégies}

L'information et la conscientisation font partie des stratégies employées par les organismes partenaires. Ce processus a permis à la communauté d'identifier la situation contre laquelle elle lutte, au nom de qui la revendication est faite et ce pour quoi elle lutte et demande un changement social (Touraine, 1965). Ampleman et collab. (1983, p. 268) indiquent que l'action « sera d'autant plus efficace qu'elle correspondra au niveau de conscience de ceux qui l'entreprennent, qu'ils pourront la contrôler réellement».

Le RÉFO, la FESFO et l'Assemblée de la francophonie de l'Ontario se sont entourés d'un comité encadreur, afin de pouvoir réajuster régulièrement la stratégie et examiner les actions à entreprendre (Comeau, 2012, p. 86). Ce comité, constitué d'étudiantes, d'élèves du secondaire, d'intellectuels, de militantes pour l'université franco-ontarienne dans les années 1980 et d'employés d'organismes communautaires (États généraux sur le postsecondaire en Ontario français, 2013b), a permis d'avoir un pouls de la communauté et de conseiller les organismes partenaires.

Finalement, ils se sont souciés de mettre de l'avant les notions d'autodétermination et de gouvernance. Ces notions sont toujours demeurées au cœur de la revendication, ce qui a permis de "donner un sens à long terme à son action, [de] joindre les réseaux d'associations et [de] faire une place aux débats de société " (Comeau, 2012, p. 86). 


\section{Les défis rencontrés (et les réponses apportées)}

\section{Financement}

Constitué depuis moins de cinq ans, le RÉFO éprouvait quelques difficultés à trouver du financement pour exécuter le mandat reçu par ses membres en 2012, les contraintes du système actuel de financement étant nombreuses. Du financement d'une durée limitée et renégociable annuellement lui était disponible, mais les bailleurs de fonds ne reconnaissaient pas la revendication comme un projet "finançable ". D'ailleurs, les dépenses de fonctionnement - salaires des cadres, assurances, espace de bureau, rencontres du conseil d'administration - ne sont pas admissibles. De plus, étant en situation minoritaire dans la province, le Regroupement affichait des résultats (chiffres) qui n'étaient souvent pas de taille, comparativement à ceux des organismes anglophones de la province.

Le RÉFO, entouré de la FESFO et de l'Assemblée de la francophonie de l'Ontario, a tout de même osé. Ensemble, ils ont tenu le lancement des États généraux sur le postsecondaire en Ontario français (mai 2013) avant même d'avoir l'entièreté du financement confirmé. Cette décision n'était pas sans conséquence. Pendant les deux années de consultations, la recherche perpétuelle de financement entreprise par l'équipe salariée et bénévole du RÉFO a demandé d'énormes sacrifices et l'a souvent empêchée de se concentrer sur d'autres aspects de sa programmation régulière.

\section{Lutte de pouvoir des institutions bilingues}

À la suite du Sommet provincial, lorsque la communauté a affirmé haut et fort le besoin d'une université gérée par et pour les francophones, les universités bilingues ont commencé à s'exprimer davantage sur la question. Elles ont tenté de minimiser l'importance du problème exposé par les organismes partenaires et ont également voulu attribuer la responsabilité du problème à une autre cause (Comeau, 2012, p. 83-84).

On n'a qu'à penser à la lettre ouverte du recteur de l'Université d'Ottawa (Rock, 2014) attaquant directement le mouvement et la réalité exprimée par les participantes et participants des États généraux :

N'en déplaise à certains, notre université a toujours été et demeure au cœur de l'épanouissement des communautés francophones, principalement dans l'Est de l'Ontario, mais aussi dans le Grand Toronto, à Windsor et bientôt, je l'espère, dans le Sud-Ouest grâce à notre projet d'expansion à Woodstock. 
Ou encore, celle du principal du Collège universitaire Glendon (Ipperciel, 2016), qui remet en question le bien-fondé de la demande de la communauté :

Le discours des universitaires de la francophonie est en général de nature pragmatique alors que celui de certains individus et groupes communautaires a tendance à être plus de l'ordre du symbolique, du rêve, des passions.

L'Université franco-ontarienne (UFO) est un projet fort louable et cohérent avec la progression sociétale des communautés francophones de l'Ontario et avec le rôle des institutions francophones dans l'expression et dans l'affirmation de son identité culturelle.

Mais entre le symbolisme et le pragmatisme, nous avons opté pour le second.

Le Consortium des universités de la francophonie ontarienne ${ }^{3}$ (CUFO) a, pour sa part, écrit directement au ministre de la Formation et des Collèges et Universités afin d'affirmer son positionnement ${ }^{4}$ en opposition au projet de création d'une université franco-ontarienne. Un des arguments mis de l'avant est la crainte de diviser le financement qui est déjà trop mince.

\section{(Non-)réponse du gouvernement}

En plus des résultats du Sommet provincial (2015), trois autres rapports ont mis de l'avant la nécessité d'une université franco-ontarienne : Pas d'avenir sans accès ${ }^{5} \mathrm{du}$ Commissariat aux services en français (2012), Bâtir l'avenir ${ }^{6}$ du Comité d'experts en matière d'éducation postsecondaire en langue française dans les régions du Centre et du Sud-Ouest de l'Ontario (2013) et Le temps d'agir! ${ }^{7}$ du Comité consultatif en matière d'éducation postsecondaire en langue française dans la région du Centre-Sud-Ouest (2016).

Malgré toutes ces recommandations, la réplique du gouvernement n'est toujours pas favorable à la mise sur pied d'une telle institution. Il opte plutôt pour le financement des institutions actuelles afin qu'elles augmentent leur offre de cours dans la région du Centre-Sud-Ouest (ministère de la Formation et des Collèges et Universités, 2013) et continue à nommer de nouveaux comités afin d'étudier la question; un conseil de planification a d'ailleurs été annoncé en juin 2016 (ministère de la Formation et des Collèges et Universités, 2016). 


\section{Changements sociaux apportés par la lutte}

Les défis n’ont pas arrêté les jeunes, les étudiantes et les étudiants. Au contraire, ces défis ont alimenté le mouvement. Shragge (2006, p. 233) élabore ce lien entre l’indignation et le militantisme :

L'organisation communautaire qui vise à réaliser le changement social commence avec cette impulsion. Apprendre quoi faire de notre colère et notre révolte, les investir dans les luttes locales, leur donner une plus grande portée et les entretenir pour alimenter notre élan en faveur de la justice sociale : tel est le défi à relever.

Lapierre et Levesque (2013, p. 57) abondent dans le même sens : « [N]ous croyons qu'un changement social d'envergure nécessite d'abord l'indignation, puis l'engagement et le militantisme. "

En réponse à la sourde oreille des institutions et à l'inaction du gouvernement, les moyens de pressions s'accumulent : demande officielle à la première ministre (Fédération de la jeunesse franco-ontarienne, 2015c), campagne de demandes d'admission symboliques à l'université franco-ontarienne (Fédération de la jeunesse franco-ontarienne, 2015b), journée de sensibilisation à Queen's Park (Fédération de la jeunesse franco-ontarienne, 2015a), journée d'action provinciale (Assemblée de la francophonie de l'Ontario, 2016a) et appel de candidatures pour la nomination d'un conseil des gouverneurs (Assemblée de la francophonie de l'Ontario, 2016b).

Bien que la lutte ne soit pas encore gagnée, des changements symboliques importants ont été occasionnés par le mouvement militant pour la création d'une université francoontarienne. Tous les conseils scolaires de langue française de l'Ontario et une vingtaine organismes communautaires ont officiellement appuyé le projet de création d'une université franco-ontarienne (États généraux sur le postsecondaire en Ontario français, 2013b).

Les médias sociaux regorgent de témoignages de membres de la communauté qui réclament une éducation en français. D'ailleurs, 8000 élèves du secondaire se sont mobilisés à travers la province le 18 février 2016, lors de la Journée d'action provinciale. Le témoignage de Marie-Pierre Héroux, une étudiante n'ayant pas pu suivre la majorité de ses cours en français à l'Université de Sudbury et à l'Université Laurentienne, est un exemple récent démontrant que la population étudiante n'a pas peur de dénoncer le statu quo et ses conséquences néfastes pour l'avenir de la communauté franco-ontarienne (Fleurent, 2016). 


\section{Conclusion}

Si le projet a une telle portée, c'est en grande partie parce qu'il est mené par et pour les jeunes, les étudiantes et les étudiants franco-ontariens, avec la communauté. Il était crucial pour les militantes et militants que le processus des États généraux soit porté par la population la plus concernée et que la question d'accès aux études postsecondaires demeure un enjeu communautaire. Le militantisme - le fait d'agir pour une cause - se trouve au cœur même du travail social et, dans le cas du mouvement pour une université franco-ontarienne, le statu quo est incubateur d'injustices et peut mener à la marginalisation du milieu étudiant francophone pauvrement desservi.

La résurgence du mouvement pour une université franco-ontarienne ne s'est pas faite $\mathrm{du}$ jour au lendemain. Face à une période que plusieurs qualifient de difficile en matière d'engagement social, les organismes partenaires ont, malgré tout, réussi à rassembler des milliers de gens sous une même cause, et ce, avec des ressources très limitées.

Les militantes et militants se sont dotés des outils nécessaires pour enclencher les changements voulus pour leur communauté. La lutte n’est pas terminée. Il a été démontré à maintes reprises que les institutions bilingues ne répondent pas aux aspirations de la société franco-ontarienne et que ces établissements sont des foyers d'assimilation (Comité consultatif en matière d'éducation postsecondaire en langue française dans la région du Centre-Sud-Ouest, 2016, p. 10; Puchala, 2015, p. 56-59; Massot, 1973, p. 139).

Il est normal que la plus grande population de francophones hors Québec en Amérique du Nord aspire à plus. La mise sur pied d'une université franco-ontarienne est une étape que l'Ontario français attend depuis longtemps. Cette institution est nécessaire au rayonnement et à la pérennité de la société franco-ontarienne.

\section{Notes}

1 La mission de l'organisme est « de défendre le droit des étudiantes et étudiants de l'Ontario français d'étudier en français dans le programme et la région de leur choix dans un contexte où elles et ils gèrent les leviers de leur éducation, afin qu'ils et elles puissent s'épanouir dans l'ensemble de la communauté franco-ontarienne et puissent y contribuer» (Regroupement étudiant francoontarien, 2014).

2 Au printemps 2012, le RÉFO apprend que le gouvernement provincial a aboli la bourse pour étudier en français. Cette bourse, d'une valeur de 1500 \$, était le seul incitatif en matière d'éducation postsecondaire de langue française. Une pétition a été lancée par le RÉFO (juillet 2012), et plus de 6000 signatures ont été recueillies. La bourse n’a pas été réinstaurée, mais une 
nouvelle subvention pour les étudiantes et étudiants qui doivent voyager plus de $80 \mathrm{~km}$ pour étudier en français a été mise en place.

3 Le CUFO regroupe l'Université d'Ottawa, l'Université Laurentienne, le Collège universitaire Glendon, l'Université Saint-Paul, l'Université de Hearst, l'Université de Sudbury et le Collège universitaire dominicain.

4 La lettre, intitulée "Positionnement du CUFO — L'avenir de l'éducation postsecondaire en français en Ontario » (19 décembre 2014), n’est plus disponible sur son site Web (http://www.cufo. $\mathrm{ca} / \#$ rapports). Le titre s'y trouve encore, mais il n'est plus possible de télécharger le fichier pdf.

5 Une section de ce rapport est dédiée au thème "Vers une université franco-ontarienne " (Commissariat aux services en français, 2012, p. 61-63).

6 Sa deuxième recommandation est la création et le démarrage de : «Un établissement d'enseignement de langue française à double vocation, universitaire et collégiale ou Deux établissements d'enseignement de langue française — un collège et une université — qui partageraient des services en commun " (Comité d'experts en matière d'éducation postsecondaire en langue française dans les régions du Centre et du Sud-Ouest de l'Ontario, 2013, p. 20).

7 Sa première recommandation est la suivante : "Que le gouvernement de l'Ontario établisse une université de langue française dans le Centre-Sud-Ouest de l'Ontario, dotée d'un campus principal dans la région du Grand Toronto (RGT) » (Comité consultatif en matière d'éducation postsecondaire en langue française dans la région du Centre-Sud-Ouest, 2016, p. 7).

\section{Bibliographie}

AMPLEMAN, Gisèle, et collab. (1983). Pratiques de conscientisation : Expériences d'éducation populaire au Québec, Montréal, Nouvelle Optique, 304 p.

ASSEMBLÉE DE LA FRANCOPHONIE DE L'ONTARIO (2016a). Fierté, impatience et résilience : la première journée d'action pour une nouvelle université franco-ontarienne connầt un franc succès!, [communiqué], réf. du 17 septembre 2017 ,

http://mesnouvellesfranco.ca/fierte-impatience-et-resilience-la-premiere-journee-daction-pourune-nouvelle-universite-franco-ontarienne-connait-un-franc-succes/

ASSEMBLÉE DE LA FRANCOPHONIE DE L'ONTARIO (2016b). Université de langue française : appel de candidatures pour la nomination d'un conseil des gouverneurs transitoire, [communiqué], réf. du 17 septembre 2017 ,

https://monassemblee.ca/universite-de-langue-francaise-appel-de-candidatures-pour-lanomination-dun-conseil-des-gouverneurs-transitoire/

COMEAU, Yvan (2012). "Les stratégies d'intervention sociopolitique favorables à l'action collective », Reflets : revue d'intervention sociale et communautaire, Vol. 18, $\mathrm{N}^{\circ} 1$, p. 61-90. 
COMITÉ CONSULTATIF EN MATIÈRE D'ÉDUCATION POSTSECONDAIRE EN LANGUE FRANÇAISE DANS LA RÉGION DU CENTRE-SUD-OUEST (2016). Le temps d'agir!, Rapport final du Comité consultatif en matière d'éducation postsecondaire en langue française dans la région du Centre-Sud-Ouest, $34 \mathrm{p}$.

COMITÉ D'EXPERTS EN MATIÈRE D'ÉDUCATION POSTSECONDAIRE EN LANGUE FRANÇAISE DANS LES RÉGIONS DU CENTRE ET DU SUD-OUEST DE L'ONTARIO (2013). Bâtir l'avenir : Pour accrô̂tre la capacité du système d'éducation en langue française dans les régions du Centre et du Sud-Ouest, [Sommaire des recommandations du Comité d'experts en matière d'éducation postsecondaire en langue française dans les régions du Centre et du SudOuest de l'Ontario], 25 p.

COMMISSARIAT AUX SERVICES EN FRANÇAIS (2012). L'état de l'éducation postsecondaire en langue française dans le Centre-Sud-Ouest de l'Ontario : Pas d'avenir sans accès, [rapport d'enquête], Toronto, $74 \mathrm{p}$.

ÉTATS GÉNÉRAUX SUR LE POSTSECONDAIRE EN ONTARIO FRANÇAIS (2015). Rapport du Sommet provincial des États généraux sur le postsecondaire en Ontario français, [Rapport synthèse du Sommet provincial d'octobre 2014], 44 p.

ÉTATS GÉNÉRAUX SUR LE POSTSECONDAIRE EN ONTARIO FRANÇAIS (2014). Rapport des consultations régionales et jeunesse des États généraux sur le postsecondaire en Ontario français, [Rapport synthèse des consultations régionales et jeunesse de l'automne 2013], 16 p.

ÉTATS GÉNÉRAUX SUR LE POSTSECONDAIRE EN ONTARIO FRANÇAIS (2013a). Accueil, réf. du 17 septembre 2017, http://etatsgeneraux.ca/

ÉTATS GÉNÉRAUX SUR LE POSTSECONDAIRE EN ONTARIO FRANÇAIS (2013b). Partenaires du projet, réf. du 17 septembre 2017, http://etatsgeneraux.ca/partenaires.php

FÉDÉRATION DE LA JEUNESSE FRANCO-ONTARIENNE (2015a). Campagne de sensibilisation auprès des député.e.s à Queen's Park pour la création d'une nouvelle université franco-ontarienne, [communiqué], réf. du 17 septembre 2017,

https://fesfo.ca/communique-campagne-de-sensibilisation-aupres-des-depute-e-s-a-queens-parkpour-la-creation-dune-nouvelle-universite-franco-ontarienne/

FÉDÉRATION DE LA JEUNESSE FRANCO-ONTARIENNE (2015b). Des milliers de personnes demandent d'être inscrites à une université gérée par et pour les francophones, [communiqué], réf. du 17 septembre 2017, https://fesfo.ca/communique-des-milliers-de-personnes-demandent-detre-inscrites-a-uneuniversite-geree-par-et-pour-les-francophones/

FÉDÉRATION DE LA JEUNESSE FRANCO-ONTARIENNE (2015c). Les francophones de l'Ontario demandent à la première ministre Wynne de s'engager à créer une université provinciale de langue française, [communiqué], réf. du 17 septembre 2017, https://fesfo.ca/universitefo-conference-1/ 
FÉDÉRATION DE LA JEUNESSE FRANCO-ONTARIENNE (2004). L'université francoontarienne : parlons impact!", [document d'opinion], 9 p.

FLEURENT, Lisa Marie (2016). "Une rentrée universitaire «all in English» à Sudbury déçoit une étudiante francophone ", Radio-Canada, réf. du 17 septembre 2017,

http://ici.radio-canada.ca/regions/ontario/2016/09/16/008-etudes-universite-sudbury-francais. shtml

GALLANT, Nicole, et Stéphanie GARNEAU (dirs.) (2016). Les jeunes et l'action politique : Participation, contestation, résistance, Québec, Presses de l'Université Laval, 262 p.

GAUTHIER, Madeleine, et Lucie PICHÉ (2001). « Participation des jeunes aux lieux d'influence et de pouvoir ", L'Action nationale, Vol. 91, № 7, p. 77-86.

IPPERCIEL, Donald (2016). " Des voix dans le désert », \#ONFR, réf. du 17 septembre 2017, http:// www5.tfo.org/onfr/des-voix-dans-le-desert

LAPIERRE, Simon, et Joscelyne LEVESQUE (2013). « 25 ans plus tard... et toujours nécessaires! Les approches structurelles dans le champ de l'intervention sociale ", Reflets : revue d'intervention sociale et communautaire, Vol. 19, № 1, p. 38-64.

LAVOIE, Jocelyne, et Jean PANET-RAYMOND (2014). La pratique de l'action communautaire, $3^{\mathrm{e}}$ édition, Québec, Presses de l'Université du Québec, 432 p.

MASSOT, Alain (1973). Dimensions de l'assimilation des étudiants francophones à l'Université d'Ottawa, [thèse de maîtrise], Ottawa, Université d'Ottawa, $232 \mathrm{p}$.

MINISTÈRE DE LA FORMATION ET DES COLLÈGES ET UNIVERSITÉS (2016). Déclaration des ministres Reza Moridi et Madeleine Meilleur sur l'élargissement de l'accès à l'éducation postsecondaire en langue française, [communiqué].

MINISTÈRE DE LA FORMATION ET DES COLLÈGES ET UNIVERSITÉS (2013). Expansion de l'éducation postsecondaire en langue française : L'Ontario prend l'engagement envers la réussite scolaire pour tous, [communiqué], réf. du 17 septembre 2017,

https://news.ontario.ca/maesd/fr/2013/10/expansion-de-leducation-postsecondaire-en-languefrancaise.html

PUCHALA, Samantha (2015). Formation universitaire en Ontario français : étude documentaire du bilinguisme à l'Université Laurentienne et ses effets sur les francophones, Sudbury, Université Laurentienne, [essai de spécialisation], $66 \mathrm{p}$.

REGROUPEMENT ÉTUDIANT FRANCO-ONTARIEN (2014). Organisation, réf. du 17 septembre 2017, http://www.refo.ca/structure.php

ROCK, Allan (2014). "L'Université d'Ottawa, l'université des Franco-Ontariens ", Le Droit, 21 octobre 2014. 
SHRAGGE, Eric (2006). Action communautaire : dérives et possibles, Montréal, Les Éditions Écosociété, $248 \mathrm{p}$.

TOURAINE, Alain (1965). Sociologie de l'action, Paris, Les Éditions du Seuil, 507 p. 